\title{
Hopf Hypersurfaces with Constant Principal Curvatures in Complex Projective or Complex Hyperbolic Spaces
}

\author{
Bang-Yen CHEN and Sadahiro MAEDA \\ Michigan State University and Shimane University \\ (Communicated by M. Oka) \\ Dedicated to Professor Hiroshi Asano on his retirement from Yokohama City University
}

\begin{abstract}
A real hypersurface of a complex space form is called a Hopf hypersurface if the characteristic vector field $\xi=-J N$ on $M$ is a principal curvature vector. The main purpose of this paper is to obtain several simple geometric characterizations of all Hopf hypersurfaces with constant principal curvatures in nonflat complex space forms.
\end{abstract}

\section{Introduction.}

It is an interesting and basic problem in differential geometry to understand hypersurfaces with constant principal curvatures in a given Riemannian manifold $\tilde{M}^{n}$. When the ambient manifold $\tilde{M}^{n}$ is a real space form $R^{n}(c)$, i.e. $R^{n}(c)=\mathbf{E}^{n}, S^{n}(c)$ or $H^{n}(c)$ (according as the curvature $c$ is zero, positive or negative), such hypersurfaces $M^{n}$ are nothing but isoparametric hypersurfaces. Although isoparametric hypersurfaces in a standard sphere have been studied extensively since 1930's by É. Cartan among others, the classification problem of isoparametric hypersurfaces in a sphere remains open (see, for instance [5], [7], [8], [11], [13], [15], [17]).

It is also very natural to consider real hypersurfaces with constant principal curvatures in a nonflat complex complex space form $\tilde{M}_{n}(c)$ with complex dimension $n$ and complex structure $J$, where $\tilde{M}_{n}(c)$ is $\mathbf{C} P^{n}(c)$ or $\mathbf{C} H^{n}(c)$ according as the holomorphic sectional curvature $c$ is positive or negative. In this respect, M. Kimura in 1986 (resp. J. Berndt in 1989) classifies real hypersurfaces $M^{2 n-1}$ in $\mathbf{C} P^{n}(c)$ (resp. in $\mathbf{C} H^{n}(c)$ ) with constant principal curvatures under the condition:

(P) For every unit normal $N$ of $M^{2 n-1}$, the vector $\xi=-J N$ is a principal curvature vector of $M^{2 n-1}$ in $\tilde{M}_{n}(c)$.

Following [4] we call a real hypersurface in $\mathbf{C} P^{n}(c)$ (or in $\mathbf{C H}^{n}(c)$ ) a Hopf hypersurface if it satisfies condition (P). Roughly speaking, Hopf hypersurfaces in $\mathbf{C} P^{n}(c)$ (resp. in $\mathrm{CH}^{n}(c)$ ) are real hypersurfaces which lie in tubes of constant radius over some complex

Received November 15, 1999 
submanifolds of $\mathbf{C} P^{n}(c)$ (resp. over some complex submanifolds or over some totally real submanifolds of $\mathrm{CH}^{n}(c)$ ) (cf. [6], [12]). These show that there exist many Hopf hypersurfaces in complex projective spaces and in complex hyperbolic spaces and that condition $(\mathrm{P})$ is natural as well.

The main purpose of this paper is to obtain some simple geometric characterizations of all Hopf hypersurfaces with constant principal curvatures in nonflat complex space forms. More precisely, in Section 2 we characterize all Hopf hypersurfaces $M$ with constant principal curvatures by using the holomorphic distribution $T^{0}(M)=\{X \in T M \mid X \perp \xi\}$ on $M$. In Section 3, we characterize such hypersurfaces by utilizing the extrinsic shape of geodesics in Hopf hypersurfaces.

Finally we remark that each real hypersurface with three constant principal curvatures in a nonflat complex space form $\tilde{M}_{n}(c), n \geqq 3$ satisfies the condition (P) (see [18], [20]). Moreover, due to their results we can see that they are nothing but homogeneous real hypersurfaces with three principal curvatures in $\tilde{M}_{n}(c)$.

\section{Auxiliary results.}

In order to prove our results we present in this section some noations, terminology and auxiliary results.

Let $M^{2 n-1}$ be an orientable real hypersurface of an $n$-dimensional nonflat complex space form $\tilde{M}_{n}(4 c)$ of constant holomorphic sectional curvature $4 c$ and $N$ a unit normal vector field on $M$. The Riemannian connections $\tilde{\nabla}$ of $\tilde{M}_{n}(4 c)$ and $\nabla$ of $M$ are related by the following formulas of Gauss and Weingarten:

$$
\begin{gathered}
\tilde{\nabla}_{X} Y=\nabla_{X} Y+\langle A X, Y\rangle N, \\
\tilde{\nabla}_{X} N=-A X,
\end{gathered}
$$

for vector fields $X$ and $Y$ tangent to $M$, where $\langle$,$\rangle denotes the Riemannian metric on M$ induced from the matric $\langle$,$\rangle on \tilde{M}_{n}(4 c)$ and $A$ denotes the shape operator of $M$ in $\tilde{M}_{n}(4 c)$. An eigenvector $X$ of the shape operator $A$ is called a principal curvature vector and an eigenvalue $\lambda$ of $A$ is called a principal curvature. We denote by $V_{\lambda}$ the eigenspace of $A$ associated with eigenvalue $\lambda$.

It is known that $M$ has an almost contact metric structure induced from the complex structure $J$ of $\tilde{M}_{n}(4 c)$, namely we have a tensor field $\phi$ of type $(1,1)$, a vector field $\xi$ and a 1-form $\eta$ on $M$ defined by

$$
\langle\phi X, Y\rangle=\langle J X, Y\rangle \quad \text { and } \quad\langle\xi, X\rangle=\eta(X)=\langle J X, N\rangle
$$

which satisfy

$$
\phi^{2} X=-X+\eta(X) \xi, \quad\langle\xi, \xi\rangle=1, \quad \phi \xi=0
$$

It follows that

$$
\left(\nabla_{X} \phi\right) Y=\eta(Y) A X-\langle A X, Y\rangle \xi
$$




$$
\nabla_{X} \xi=\phi A X
$$

Let $R$ be the curvature tensor of $M$. We have the equations of Gauss and Codazzi given by:

$$
\begin{aligned}
&\langle R(X, Y) Z, W\rangle= c\{\langle Y, Z\rangle\langle X, W\rangle-\langle X, Z\rangle\langle Y, W\rangle+\langle\phi Y, Z\rangle\langle\phi X, W\rangle \\
&-\langle\phi X, Z\rangle\langle\phi Y, W\rangle-2\langle\phi X, Y\rangle\langle\phi Z, W\rangle\} \\
&+\langle A Y, Z\rangle\langle A X, W\rangle-\langle A X, Z\rangle\langle A Y, W\rangle, \\
&\left(\nabla_{X} A\right) Y-\left(\nabla_{Y} A\right) X=c\{\eta(X) \phi Y-\eta(Y) \phi X-2\langle\phi X, Y\rangle \xi\} .
\end{aligned}
$$

We recall the following classification theorems on Hopf hypersurfaces with constant principal curvatures in nonflat complex space forms.

THEOREM A ([9], [19]). Let $M^{2 n-1}$ be a Hopf hypersurface of $\mathbf{C} P^{n}(n \geqq 2)$ with constant principal curvatures. Then $M$ is locally congruent to one of the following:

$\left(A_{1}\right) \quad$ a tube of radius $r$ over hyperplane $\mathbf{C} P^{n-1}$, where $0<r<\pi / 2$;

$\left(A_{2}\right)$ a tube of radius $r$ over totally geodesic $\mathbf{C} P^{k}(1 \leqq k \leqq n-2)$, where $0<r<$ $\pi / 2$

(B) a tube of radius $r$ over complex hyperquadric $\mathbf{C} Q^{n-1}$, where $0<r<\pi / 4$;

(C) a tube of radius $r$ over $\mathbf{C} P^{1} \times \mathbf{C P} P^{(n-1) / 2}$, where $0<r<\pi / 4$ and $n(\geqq 5)$ is odd;

(D) a tube of radius $r$ over complex Grassmann $\mathbf{C G}_{2,5}$, where $0<r<\pi / 4$ and $n=9$;

(E) a tube of radius $r$ over Hermitian symmetric space $S O(10) / U(5)$, where $0<$ $r<\pi / 4$ and $n=15$.

THEOREM B ([4]). Let $M^{2 n-1}$ be a Hopf hypersurface of $\mathrm{CH}^{n}(n \geqq 2)$ with constant principal curvatures. Then $M$ is locally congruent to one of the following:

$\left(A_{0}\right)$ a horosphere in $\mathbf{C} H^{n}$;

$\left(A_{1}\right)$ a tube of radius $r$ over $\mathbf{C} H^{k}(k=0, n-1)$, where $0<r<\infty$;

$\left(A_{2}\right)$ a tube of radius $r$ over $\mathbf{C} H^{k}(1 \leqq k \leqq n-2)$, where $0<r<\infty$;

(B) a tube of radius $r$ over $\mathbf{R} H^{n}$, where $0<r<\infty$.

The following proposition is fundamental concerning Hopf hypersurfaces in nonflat complex space forms (cf. [14, pp. 244-245]).

PROPOSITION A. Let $M^{2 n-1}(n \geqq 2)$ be a Hopf hypersurface in a nonflat complex space form $\tilde{M}_{n}(4 c)$ and $\alpha$ the principal curvature corresponding to the characteristic vector field $\xi$. We have

(1) $\alpha$ is locally constant.

(2) If $X$ is a tangent vector of $M$ perpendicular to $\xi$ with $A X=\lambda X$, then $(2 \lambda-$ $\alpha) A \phi X=(\alpha \lambda+2 c) \phi X$. In particular, we have $A \phi X=\frac{\alpha \lambda+2 c}{2 \lambda-\alpha} \phi X$ whenever $c>0$. 
We simply call real hypersurfaces of type $\left(A_{0}\right),\left(A_{1}\right)$ and $\left(A_{2}\right)$ given in the lists of Theorem A and Theorem B type A hypersurfaces. The following result is a well-known characterization of type $A$ hypersurfaces (cf. [14, p. 264]).

PROPOSITION B. Let $M^{2 n-1}(n \geqq 2)$ be a real hypersurface of a nonflat complex space form $\tilde{M}_{n}(4 c)$. Then the following three conditions are mutually equivalent:

(1) $M$ is locally congruent to a type A hypersurface.

(2) $\phi A=A \phi$, where $\phi$ is the structure tensor and $A$ is the shape operator of $M$ in $\tilde{M}_{n}(4 c)$.

(3) The covariant derivative of the shape operator $A$ of $M$ in $\tilde{M}_{n}(4 c)$ satisfies $\left(\nabla_{X} A\right) Y$ $=-c(\langle\phi X, Y\rangle \xi+\eta(Y) \phi X)$.

\section{Holomorphic distribution of Hopf hypersurfaces.}

First we prove the following general result.

PROPOSITION 1. For every Hopf hypersurface $M^{2 n-1}$ in a nonflat complex space form, the holomorphic distribution $T^{0} M=:\{X \in T M \mid X \perp \xi\}$ is non-integrable.

Proof. Suppose that $T^{0} M$ is integrable. Then Proposition 5 of [11] implies

$$
\langle(\phi A+A \phi) X, Y\rangle=0 \text { for any } X, Y \in T^{0} M .
$$

This, together with the hypothesis that $\xi$ is principal, yields $\phi A+A \phi \equiv 0$ on $M$, which is a contradiction (see $[14$, p. 252]).

Proposition 1 implies immediately that $T^{0} M$ is not integrable for every hypersurface $M$ given in the lists of Theorems A and B. However every Hopf hypersurface listed in Theorems $\mathrm{A}$ and $\mathrm{B}$ also satisfies the following decomposition condition:

CONDITION $(D): \quad T^{0} M$ can be decomposed as the direct sum of principal foliations $V_{\lambda_{i}}$ 's of $M$ in $\tilde{M}_{n}(4 c)$.

In this section we investigate the holomorphic distributions of Hopf hypersurfaces in details. In particular, we study hypersurfaces in nonflat complex space forms which satisfy condition (D); thus, $T^{0} M=\sum_{i=1}^{g} V_{\lambda_{i}}$, where $V_{\lambda_{i}}=\left\{X \in T^{0} M \mid A X=\lambda_{i} X\right\}$ and $g$ denotes the number of distinct principal curvatures in the directions orthogonal to $\xi$. Needless to say, $(D)$ always implies $(P)$. Moreover, on the open dense subset $\mathcal{U}$ of $M$ defined by

$$
\begin{aligned}
\mathcal{U}= & \{p \in M \mid \text { the multiplicity of each principal curvature of } \\
& \left.M \text { in } \tilde{M}_{n}(4 c) \text { is constant on some neighborhood of } p\right\},
\end{aligned}
$$

these two conditions are equivalent locally. In this sense, in local theory condition $(P)$ and condition $(D)$ are equivalent almost everywhere on $M$.

In order to establish our main results we also need the following result which characterizes type $B$ hypersurfaces given in the lists of Theorems $\mathrm{A}$ and $\mathrm{B}$ for $n=2$. 
Proposition 2. Let $M$ be a real hypersurface of $\tilde{M}_{2}(4 c)$. If $T^{0} M=V_{\lambda_{1}} \oplus V_{\lambda_{2}}$ with $\lambda_{1} \neq \lambda_{2}$ and $\operatorname{dim} V_{\lambda_{1}}=\operatorname{dim} V_{\lambda_{2}}=1$ and if the integrable curves of $V_{\lambda_{1}}$ and $V_{\lambda_{2}}$ are geodesics in $M$, then $\lambda_{1}$ and $\lambda_{2}$ are both locally constant on $M$.

Proof. Let $\left\{e_{1}, e_{2}, \xi\right\}$ be a local field of orthonormal frames in $M$ in such a way that $e_{i} \in V_{\lambda_{i}}(i=1,2)$ and $e_{2}=\phi e_{1}$. We first note that $\xi$ is principal, because $\left\langle A \xi, e_{i}\right\rangle=$ $\lambda_{i}\left\langle\xi, e_{i}\right\rangle=0(i=1,2)$. So it follows from (1.3) and (1.5) that $\nabla_{\xi} \xi=0$. By hypothesis we also have $\nabla_{e_{1}} e_{1}=\nabla_{e_{2}} e_{2}=0$. From (1.5) we obtain

$$
\nabla_{e_{1}} \xi=\lambda_{1} e_{2}, \quad \nabla_{e_{2}} \xi=-\lambda_{2} e_{1}, \quad \nabla_{e_{1}} e_{2}=-\lambda_{1} \xi, \quad \nabla_{e_{2}} e_{1}=\lambda_{2} \xi
$$

Equation (1.7) of Codazzi implies

$$
\left(\nabla_{e_{1}} A\right) e_{2}-\left(\nabla_{e_{2}} A\right) e_{1}=-2 c \xi
$$

On the other hand, (2.1) yields

$$
\begin{aligned}
\left(\nabla_{e_{1}} A\right) e_{2}-\left(\nabla_{e_{2}} A\right) e_{1} & =\nabla_{e_{1}}\left(A e_{2}\right)-A \nabla_{e_{1}} e_{2}-\nabla_{e_{2}}\left(A e_{1}\right)+A \nabla_{e_{2}} e_{1} \\
& =\left(e_{1} \lambda_{2}\right) e_{2}+\left(\lambda_{2} I-A\right) \nabla_{e_{1}} e_{2}-\left(e_{2} \lambda_{1}\right) e_{1}-\left(\lambda_{1} I-A\right) \nabla_{e_{2}} e_{1} \\
& =-\left(e_{2} \lambda_{1}\right) e_{1}+\left(e_{1} \lambda_{2}\right) e_{2}+\left\{\alpha\left(\lambda_{1}+\lambda_{2}\right)-2 \lambda_{1} \lambda_{2}\right\} \xi .
\end{aligned}
$$

These two equations imply the following three relations:

$$
\begin{aligned}
-2 c & =\alpha\left(\lambda_{1}+\lambda_{2}\right)-2 \lambda_{1} \lambda_{2} . \\
e_{2} \lambda_{1} & =0 . \\
e_{1} \lambda_{2} & =0 .
\end{aligned}
$$

Since $\alpha$ is locally constant, (2.2) and (2.3) imply

$$
\left(\alpha-2 \lambda_{1}\right)\left(e_{2} \lambda_{2}\right)=0 .
$$

Similarly, (2.2) and (2.4) imply

$$
\left(\alpha-2 \lambda_{2}\right)\left(e_{1} \lambda_{1}\right)=0 .
$$

Now assume $c=-1$. It suffices to consider the following three cases.

Case $(i): \quad \alpha \equiv 2 \lambda_{2}$ locally and $\alpha \neq 2 \lambda_{1}$ at some point $p \in M$. In this case, (2.2) yields $\left(\lambda_{2}\right)^{2}=1$. Without loss of generality, we may assume $\lambda_{2}=1$ and hence $\alpha=2$. By putting $\lambda_{1}=\lambda$, we have

$$
\left\{\begin{array}{l}
\nabla_{e_{1}} e_{1}=\nabla_{e_{2}} e_{2}=\nabla_{\xi} \xi=0 \\
\nabla_{e_{1}} e_{2}=-\lambda \xi, \quad \nabla_{e_{2}} e_{1}=\xi \\
\nabla_{e_{1}} \xi=\lambda e_{2}, \quad \nabla_{e_{2}} \xi=-e_{1}
\end{array}\right.
$$

We note that $\lambda \neq 1$ on some neighborhood $\mathcal{V}$ of $p$, due to the continuity of $\lambda$. By putting $\nabla_{\xi} e_{1}=\mu e_{2}$ we obtain from (1.7) that

$$
\left(\nabla_{e_{1}} A\right) \xi-\left(\nabla_{\xi} A\right) e_{1}=e_{2}
$$


On the other hand, we have

$$
\begin{aligned}
\left(\nabla_{e_{1}} A\right) \xi-\left(\nabla_{\xi} A\right) e_{1} & =2\left(\nabla_{e_{1}} \xi\right)-A\left(\nabla_{e_{1}} \xi\right)-\nabla_{\xi}\left(\lambda e_{1}\right)+A\left(\nabla_{\xi} e_{1}\right) \\
& =\lambda e_{2}-(\xi \lambda) e_{1}-\lambda \mu e_{2}+\mu e_{2} .
\end{aligned}
$$

These two equations imply $\mu=1$ on $\mathcal{V}$, so we get $\nabla_{\xi} e_{1}=e_{2}$ and hence $\nabla_{\xi} e_{2}=-e_{1}$.

Let $R$ and $\tilde{R}$ denote the Riemannian curvature tensors of $M$ and $\mathrm{CH}^{2}(-4)$, respectively. Then we obtain

$$
\begin{aligned}
\left\langle R\left(e_{1}, e_{2}\right) e_{2}, e_{1}\right\rangle & =\lambda\left\langle\nabla_{e_{2}} \xi, e_{1}\right\rangle+\lambda\left\langle\nabla_{\xi} e_{2}, e_{1}\right\rangle+\left\langle\nabla_{\xi} e_{2}, e_{1}\right\rangle \\
& =-2 \lambda-1
\end{aligned}
$$

On the other hand, from the Gauss equation we find

$$
\begin{aligned}
\left\langle R\left(e_{1}, e_{2}\right) e_{2}, e_{1}\right\rangle & =\left\langle\tilde{R}\left(e_{1}, e_{2}\right) e_{2}, e_{1}\right\rangle+\left\langle A e_{1}, e_{1}\right\rangle\left\langle A e_{2}, e_{2}\right\rangle-\left\langle A e_{1}, e_{2}\right\rangle^{2} \\
& =-4+\lambda .
\end{aligned}
$$

Thus $\lambda=1$ on $\mathcal{V}$, which is a contradiction. Hence, case $(i)$ cannnot occur.

Case (ii): $\alpha \equiv 2 \lambda_{1}$ locally and $\alpha \neq 2 \lambda_{2}$ at some point $p \in M$. This case cannot occur due to the same reasoning as in Case $(i)$.

Case (iii): $\alpha \neq 2 \lambda_{1}$ and $\alpha \neq 2 \lambda_{2}$ at some point $p \in M$. In this case, equations (2.3), (2.4), (2.5), (2.6) yield

$$
e_{1} \lambda_{2}=e_{1} \lambda_{2}=e_{2} \lambda_{1}=e_{2} \lambda_{2}=0
$$

on some neighborhood $\mathcal{V}$ of the point $p$.

From (1.7) we have

$$
\left(\nabla_{e_{1}} A\right) \xi-\left(\nabla_{\xi} A\right) e_{1}=e_{2} .
$$

On the other hand, we also have

$$
\begin{aligned}
\left(\nabla_{e_{1}} A\right) \xi-\left(\nabla_{\xi} A\right) e_{1} & =\nabla_{e_{1}}(A \xi)-A\left(\nabla_{e_{1}} \xi\right)-\nabla_{\xi}\left(A e_{1}\right)+A\left(\nabla_{\xi} e_{1}\right) \\
& =\alpha \lambda_{1} e_{2}-\lambda_{1} \lambda_{2} e_{2}-\left(\xi \lambda_{1}\right) e_{1}-\left(\lambda_{1} I-A\right)\left(\nabla_{\xi} e_{1}\right) .
\end{aligned}
$$

Since $\left(\lambda_{1} I-A\right)\left(\nabla_{\xi} e_{1}\right)$ is perpendicular to $e_{1}$, these two equations imply $\xi \lambda_{1}=0$. Similarly, we also have $\xi \lambda_{2}=0$. Hence, both $\lambda_{1}$ and $\lambda_{2}$ are locally constant.

Finally, we consider the case of $c=1$. Note that $2 \lambda-\alpha \neq 0$ for each principal curvature $\lambda$ in the direction orthogonal to $\xi$ (see Proposition A). Therefore by applying the same discussion as in Case (iii) we can conclude that both $\lambda_{1}$ and $\lambda_{2}$ are locally constant.

We are now in a position to prove the following theorem. In doctoral thesis "Uber Untermanningfaltigkeiten von komplexen Raumformen" (Koln, 1989), Berndt proved one direction of the following theorem, namely he proved the integrability of the distribution for Hopf hypersurfaces with constant principal curvatures. However we here write the proof of the following theorem in detail for readers. The authors are grateful to Professor L. Vanhecke for his information about the doctoral thesis. 
THEOREM 1. Let $M$ be a real hypersurface of $\mathbf{C} H^{n}$ with $n \geqq 2$. Suppose that the holomorphic distribution $T^{0} M$ satisfies condition $(D)$. Then $M$ is a Hopf hypersurface with constant principal curvatures if and only if $T^{0} M$ satisfies one of the following two conditions:

(i) The distribution $V_{\lambda_{i}} \otimes\{\xi\}_{\mathbf{R}}$ is integrable for every principal foliation $V_{\lambda_{i}}$ of $T^{0} M$.

(ii) Every principal foliation $V_{\lambda_{i}}$ of $T^{0} M$ is integrable and every leaf of $V_{\lambda_{i}}$ is a totally geodesic submanifold of $M$.

PROOF. $\quad \Leftrightarrow \quad$ First we prove that the holomorphic distribution of every type $A$ hypersurface satisfies condition (i). Since this is obvious for type $A_{0}$ and type $A_{1}$ hypersurfaces, so we assume $M$ is of type $A_{2}$, and thus $T^{0} M$ is decomposed as $T^{0} M=V_{\tanh (r)} \oplus V_{\operatorname{coth}(r)}$ (cf. [4]). Notice that

$$
A \xi=2 \operatorname{coth}(2 r) \xi, \quad \phi\left(V_{\tanh (r)}\right)=V_{\tanh (r)}, \quad \phi\left(V_{\operatorname{coth}(r)}\right)=V_{\operatorname{coth}(r)} .
$$

Our aim here is to verify that both $V_{\tanh (r)} \oplus\{\xi\}_{\mathbf{R}}$ and $V_{\operatorname{coth}(r)} \oplus\{\xi\}_{\mathbf{R}}$ are integrable and that the leaves of $V_{\tanh (r)} \oplus\{\xi\}_{\mathbf{R}}$ and $V_{\operatorname{coth}(r)} \oplus\{\xi\}_{\mathbf{R}}$ are totally geodesic submanifolds of $\boldsymbol{M}$.

Put $\mathfrak{T}=V_{\tanh (r)} \oplus\{\xi\}_{\mathbf{R}}$. We claim the following.

$$
\nabla_{\xi} \xi \in \mathfrak{T}, \quad \nabla_{X} \xi \in \mathfrak{T}, \quad \nabla_{\xi} X \in \mathfrak{T} \quad \text { and } \quad \nabla_{X} Y \in \mathfrak{T}, \quad \forall X, Y \in V_{\tanh (r)} .
$$

The first two assertions hold, since $\nabla_{\xi} \xi=0$ and $\nabla_{X} \xi=\phi A X=\tanh (r) \phi X \in V_{\tanh (r)}$.

Next, we observe that

$$
\begin{aligned}
\left(\nabla_{\xi} A\right) X-\left(\nabla_{X} A\right) \xi & =\nabla_{\xi}(A X)-A \nabla_{\xi} X-\nabla_{X}(A \xi)+A \nabla_{X} \xi \\
& =(\tanh (r) I-A) \nabla_{\xi} X-\alpha \phi A X+A \phi A X \\
& =(\tanh (r) I-A) \nabla_{\xi} X+\tanh (r)(\tanh (r)-\alpha) \phi X .
\end{aligned}
$$

On the other hand, from (1.7) we also have $\left(\nabla_{\xi} A\right) X-\left(\nabla_{X} A\right) \xi=-\phi X \in V_{\tanh (r)}$. Thus, for any $Z \in V_{\lambda}(\lambda \neq \tanh (r))$, we find $\left\langle(\tanh (r) I-A) \nabla_{\xi} X, Z\right\rangle=0$, and hence $\nabla_{\xi} X \in$ $V_{\tanh (r)} \subset \mathfrak{T}$. This proves the third assertion.

Finally, for $X, Y \in V_{\tanh (r)}$ and $Z \in V_{\operatorname{coth}(r)}$, we have

$$
\begin{aligned}
\left\langle\left(\nabla_{X} A\right) Y, Z\right\rangle & =\left\langle\nabla_{X}(A Y)-A \nabla_{X} Y, Z\right\rangle \\
& =\left\langle(\tanh (r) I-A) \nabla_{X} Y, Z\right\rangle \\
& =(\tanh (r)-\operatorname{coth}(r))\left\langle\nabla_{X} Y, Z\right\rangle .
\end{aligned}
$$

On the other hand, since $A$ is symmetric, we obtain from $\langle\phi X, Z\rangle=0$ and equation (1.7) of Codazzi that

$$
\begin{aligned}
\left\langle\left(\nabla_{X} A\right) Y, Z\right\rangle & =\left\langle\left(\nabla_{X} A\right) Z, Y\right\rangle \\
& =\left\langle\left(\nabla_{Z} A\right) X, Y\right\rangle \\
& =\left\langle\nabla_{Z}(A X)-A \nabla_{Z} X, Y\right\rangle \\
& =\left\langle(\tanh (r) I-A) \nabla_{Z} X, Y\right\rangle \\
& =\left\langle\nabla_{Z} X,(\tanh (r) I-A) Y\right\rangle=0 .
\end{aligned}
$$


These two formulas imply the fourth assertion: $\nabla_{X} Y \in \mathfrak{T}$, $\operatorname{since} \tanh (r)-\operatorname{coth}(r) \neq 0$. Consequently, the distribution $V_{\tanh (r)} \oplus\{\xi\}_{\mathbf{R}}$ is integrable (and moreover each leaf of $V_{\tanh (r)} \oplus\{\xi\}_{\mathbf{R}}$ is a totally geodesic submanifold of $M$ ). A similar argument implies that $V_{\operatorname{coth}(r)} \oplus\{\xi\}_{\mathbf{R}}$ is also integrable.

Now, assume $M$ is of type $B$. Then $T^{0} M$ is decomposed as: $T^{0} M=V_{\tanh (r)} \oplus V_{\operatorname{coth}(r)}$. Notice that in this case we have $A \xi=2 \tanh (2 r) \xi$ and $\phi V_{\tanh (r)}=V_{\operatorname{coth}(r)}$. We shall prove that $T^{0} M$ satisfies condition (ii).

First we claim that $\nabla_{X} Y \in V_{\tanh (r)}$, for $X, Y$ in $V_{\tanh (r)}$. This can be proved as follows: For $X, Y$ in $V_{\tanh (r)}$, we have

$$
A \nabla_{X} Y=\nabla_{X}(A Y)-\left(\nabla_{X} A\right) Y=\tanh (r) \nabla_{X} Y-\left(\nabla_{X} A\right) Y .
$$

Since $\langle\phi X, Y\rangle=0$ and $A$ is symmetric, equation (1.7) implies, for any $Z \in T M$, that

$$
\begin{aligned}
\left\langle\left(\nabla_{X} A\right) Y, Z\right\rangle & \left.=\left\langle\left(\nabla_{X} A\right) Z, Y\right) \quad \text { (by the symmetry of } A\right) \\
& \left.=\left\langle\left(\nabla_{Z} A\right) X, Y\right\rangle \quad \text { (by equation }(1.7)\right) \\
& =\left\langle\nabla_{Z}(A X)-A \nabla_{Z} X, Y\right\rangle \\
& =\left\langle(\tanh (r) I-A) \nabla_{Z} X, Y\right\rangle=0 .
\end{aligned}
$$

These two equations show that $A\left(\nabla_{X} Y\right)=\tanh (r) \nabla_{X} Y$ for any $X, Y \in V_{\tanh (r)}$. Hence, $V_{\tanh (r)}$ is integrable and every leaf $L_{\tanh (r)}$ of the principal foliation $V_{\tanh (r)}$ is a totally geodesic submanifold of $M$. (In fact, the leaf $L_{\tanh (r)}$ is a totally umbilic hypersurface of constant curvature $c$ with $\sqrt{c+1}=\tanh (r)$ in $\mathbf{R} H^{n}(-1)$, and $\mathbf{R} H^{n}(-1)$ is imbedded as a totally real totally geodesic submanifold in $\left.\mathrm{CH}^{n}(-4)\right)$. Similar results also hold for $V_{\operatorname{coth}(r)}$ by applying similar arguments.

$(\Leftarrow)$ Suppose that $T^{0} M$ satisfies the condition (i). Then, for any $X$ in $V_{\lambda_{i}}$, we have $\nabla_{x} \xi-\nabla_{\xi} X \in V_{\lambda_{i}} \oplus\{\xi\}_{\mathbf{R}}$. Since $\xi$ is principal, we also have $\left\langle\nabla_{\xi} X, \xi\right\rangle=-\left\langle X, \tilde{\nabla}_{\xi} \xi\right\rangle=$ $\langle X, J A \xi\rangle=0$ for any $X$ in $V_{\lambda_{i}}$. These show that, for any $X$ in $V_{\lambda_{i}}, \nabla_{X} \xi-\nabla_{\xi} X$ is perpendicular to $\xi$. Hence, $\nabla_{X} \xi-\nabla_{\xi} X$ lies in $V_{\lambda_{i}}$. Therefore

$$
A\left(\nabla_{X} \xi-\nabla_{\xi} X\right)=\lambda_{i}\left(\nabla_{X} \xi-\nabla_{\xi} X\right) \text { for } \forall X \in V_{\lambda_{i}} \text {. }
$$

Now, we consider the case that $2 \lambda_{i}-\alpha \neq 0$ on some neighborhood of an arbitrary fixed point $p$. It folllows from (2.7) and Proposition A that

$$
\left(A-\lambda_{i} I\right) \nabla_{\xi} X=\lambda_{i}\left(\frac{\alpha \lambda_{i}-2}{2 \lambda_{i}-\alpha}-\lambda_{i}\right) \phi X .
$$

Applying (1.5) and (2.8) we obtain

$$
\begin{aligned}
\left(\nabla_{X} A\right) \xi-\left(\nabla_{\xi} A\right) X & =\nabla_{X}(\alpha \xi)-A \nabla_{X} \xi-\nabla_{\xi}(A X)+A \nabla_{\xi} X \\
& =\lambda_{i}\left(\alpha-\lambda_{i}\right) \phi X-\left(\xi \lambda_{i}\right) X .
\end{aligned}
$$

On the other hand, we obtain from (1.7) that

$$
\left(\nabla_{X} A\right) \xi-\left(\nabla_{\xi} A\right) X=\phi X .
$$


These two equations imply that the principal curvature $\lambda_{i}$ is a solution of the quadratic equation:

$$
\lambda_{i}^{2}-\alpha \lambda_{i}+1=0
$$

which implies that $\lambda_{i}$ is constant.

Next, consider the case that $2 \lambda_{i}-\alpha=0$ at some point $p$. In this case, the discussion above for the case of $2 \lambda_{i}-\alpha \neq 0$ tells us that $2 \lambda_{i}-\alpha=0$ holds on some neighborhood of $p$. Thus, each principal curvature of $M$ is locally constant. Notice that $M$ is not of type $B$, since each principal curvature $\lambda_{i}$ of a type $B$ hypersurface satisfies neither (2.9) nor $2 \lambda_{i}-\alpha=0$.

Next, suppose that $T^{0} M$ satisfies the condition (ii). Then, for any $X, Y \in V_{\lambda_{i}}$, we have $A \nabla_{X} Y=\lambda_{i} \nabla_{X} Y$; and hence $\left(\nabla_{X} A\right) Y=\left(X \lambda_{i}\right) Y$.

We divide our discussion into two cases.

Case (a): $\quad \operatorname{dim} V_{\lambda_{i}} \geqq 2$. In this case we have

$$
\left(\nabla_{X} A\right) Y-\left(\nabla_{Y} A\right) X=\left(X \lambda_{i}\right) Y-\left(Y \lambda_{i}\right) X \quad \text { for } \forall X, Y \in V_{\lambda_{i}} .
$$

On the other hand, from (1.7) we also have

$$
\left(\nabla_{X} A\right) Y-\left(\nabla_{Y} A\right) X=2\langle\phi X, Y\rangle \xi \quad \text { for } \forall X, Y \in V_{\lambda_{i}} .
$$

By choosing $X, Y$ arbitrary two independent vectors in $V_{\lambda_{i}}$, we obtain from these two equations that $X \lambda_{i}=Y \lambda_{i}=\langle\phi X, Y\rangle=\left(\nabla_{X} A\right) Y=0$. Hence, by applying continuity, we obtain

$$
\left(\nabla_{X} A\right) Y=\langle\phi X, Y\rangle=0, \quad \text { for } \forall X, Y \in V_{\lambda_{i}} \text {. }
$$

Therefore, for any unit vector $X \in V_{\lambda_{i}}$ and any $Z \in T M$, we obtain from (1.7) and (2.10) that

$$
\begin{aligned}
0 & =\left\langle\left(\nabla_{X} A\right) X, Z\right\rangle \\
& =\left\langle\left(\nabla_{X} A\right) Z, X\right\rangle \\
& =\left\langle\left(\nabla_{Z} A\right) X, X\right\rangle \\
& =\left\langle\nabla_{Z}(A X)-A \nabla_{Z} X, X\right\rangle \\
& =\left\langle\left(Z \lambda_{i}\right) X+\left(\lambda_{i} I-A\right) \nabla_{Z} X, X\right\rangle \\
& =Z \lambda_{i} .
\end{aligned}
$$

So, $\lambda$ is constant.

Case (b): $\operatorname{dim} V_{\lambda_{i}}=1$. Since $\alpha$ is constant by Proposition A, we only need to consider the case that $2 \lambda_{i}-\alpha \neq 0$ on some neighborhood of an arbitrary fixed point $p$.

Let $e$ be a unit vector in $V_{\lambda_{i}}$ so that $A e=\lambda_{i} e$. Then Proposition A implies $A \phi e=$ $\frac{\alpha \lambda_{i}-2}{2 \lambda_{i}-\alpha} \phi e$. Hence, $\phi e \in V_{\lambda_{j}}$ for some $j$ with $\lambda_{j}=\frac{\alpha \lambda_{i}-2}{2 \lambda_{i}-\alpha} \neq \lambda_{i}$. When $\operatorname{dim} V_{\lambda_{j}} \geqq 2$, Case (a) implies that $\lambda_{j}$ is constant, and thus $\lambda_{i}$ is also constant. Therefore, this case reduces to the case: $\operatorname{dim} V_{\lambda_{i}}=\operatorname{dim} V_{\lambda_{j}}=1$.

We put $\mathfrak{T}=\{\xi, e, \phi e\}_{\mathbf{R}}$ with $A e=\lambda e$ and $A \phi e=\frac{\alpha \lambda-2}{2 \lambda-\alpha} \phi e$. For simplicity we denote $\frac{\alpha \lambda-2}{2 \lambda-\alpha}$ by $\mu$. It follows from $\operatorname{dim} V_{\lambda}=1$ that $\lambda \neq \mu$. Now, we prove that $\mathfrak{T}$ is integrable and each leaf of $\mathfrak{T}$ is a totally geodesic submanifold of the real hypersurface $M$. 
First, notice that $\nabla_{e} e=\nabla_{\phi e} \phi e=0$, since both $\{e\}_{\mathbf{R}}$ and $\{\phi e\}_{\mathbf{R}}$ satisfy condition (ii) of Theorem 1. Also, it is easy to verify that $\nabla_{\xi} \xi \in \mathfrak{T}, \nabla_{e} \xi \in \mathfrak{T}$ and $\nabla_{e}(\phi e) \in \mathfrak{T}$. Now, we prove $\nabla_{\xi} e \in \mathfrak{T}$. For this, we observe that

$$
\begin{aligned}
\left(\nabla_{\xi} A\right) e-\left(\nabla_{e} A\right) \xi & =\nabla_{\xi}(A e)-A \nabla_{\xi} e-\nabla_{e}(A \xi)+A \nabla_{e} \xi \\
& =(\xi \lambda) e+(\lambda I-A) \nabla_{\xi} e-\alpha \lambda \phi e+\lambda \mu \phi e .
\end{aligned}
$$

On the other hand, equation (1.7) of Codazzi gives

$$
\left(\nabla_{\xi} A\right) e-\left(\nabla_{e} A\right) \xi=-\phi e .
$$

Thus, we find

$$
(\lambda I-A) \nabla_{\xi} e=-\{\lambda(\mu-\alpha)+1\} \phi e .
$$

Since $\lambda$ is different from other eigenvalues of $A$ and $\nabla_{\xi} e$ is perpendicular to $V_{\lambda}$, the last equation implies that $\nabla_{\xi} e \in\{\phi e\}_{\mathbf{R}} \subset \mathfrak{T}$. Similarly, we have $\nabla_{\xi}(\phi e) \in \mathfrak{T}$.

Next we prove $\nabla_{\phi e} e \in \mathfrak{T}$. From $A e=\lambda e$ and $A(\phi e)=\mu \phi e$, we have

$$
\left(\nabla_{e} A\right) \phi e-\left(\nabla_{\phi e} A\right) e=(e \mu) \phi e+(\mu I-A) \nabla_{e}(\phi e)-\phi e(\lambda) \cdot e-(\lambda I-A) \nabla_{\phi e} e .
$$

From (1.4) and $\nabla_{e} e=0$, we also have

$$
(\mu I-A) \nabla_{e}(\phi e)=-\lambda(\mu-\alpha) \xi .
$$

On the other hand, equation (1.7) gives

$$
\left(\nabla_{e} A\right) \phi e-\left(\nabla_{\phi e} A\right) e=2 \xi .
$$

The three equations above yield

$$
2 \xi=(e \mu) \phi e-\lambda(\mu-\alpha) \xi-\phi e(\lambda) \cdot e-(\lambda I-A) \nabla_{\phi e} e,
$$

which implies $\nabla_{\phi e} e \in\{\xi, \phi e\}_{\mathbf{R}} \subset \mathfrak{T}$. Consequently, $\mathfrak{T}$ is an integrable distribution and its leaves are totally geodesic submanifolds of $M$.

Assume $B$ is one of the leaves of $\mathfrak{T}$. Then $B$ is totally geodesic in the real hypersurface $M$ with $T B=\{e, \phi e, \xi\}_{\mathbf{R}}$. Let $N$ denote the normal bundle of $B$ in $M$. Then $N$ is invariant under the action of $J$ and it can be considered as the subbundle of the normal bundle $T^{\perp} B$ of $B$ in $C H^{n}(-4)$. Since $B$ is totally geodesic in $M$, we have $\nabla_{X} Z \in N$ for any $X \in T B$ and $Z \in N$. Moreover, since $e, \phi e$ and $\xi$ are principal vectors of $M$, we also have $\langle A X, Z\rangle=0$. Therefore, $\tilde{\nabla}_{X} Z \in N$ for any $X \in T B$ and $Z \in N$. These show that $N$ is a holomorphic normal subbundle over $B$ which is parallel in $T^{\perp} B$ and, moreover, the first normal spaces of $B$ in $\mathbf{C H}^{n}(-4)$ lie in $\{J \xi\}_{\mathbf{R}}$, which is also a parallel subbundle of $T^{\perp} B$ (due to the fact that $\{J \xi\}_{\mathbf{R}}$ is the orthogonal complement of $N$ in $T^{\perp} B$ ). Hence, by reduction theorem, $B$ is a real hypersurface of a totally geodesic complex submanifold $\mathrm{CH}^{2}(-4)$ of $\mathbf{C} H^{n}(-4)$. Because $B$ satisfies the hypothesis of Proposition $2, \lambda$ must be locally constant along $B$, which implies $\left(\nabla_{e} A\right) e=0$ along $B$. Therefore, we may apply the same computation as in (2.11) to obtain $Z \lambda=0$ on $M$ for any $Z$ in $T M$. Consequently, the principal curvature $\lambda$ associated with $e$ is constant too. By virtue of our argument we know that $M$ is locally congruent to a type $B$ hypersurface. 
Here we consider the list of Theorem A. Needless to say, the holomorphic distribution $T^{0} M$ of a type A hypersurface $M$ (resp. a type B hypersurface $M$ ) satisfies the condition (i) (resp. the condition (ii)) of Theorem 1 . Let $M$ be of type C, type $\mathrm{D}$ or type $\mathrm{E}$ which is a tube of radius $r(0<r<\pi / 4)$. Then $T^{0} M$ is decomposed as (see [19]):

$$
T^{0} M=V_{\lambda_{1}} \oplus V_{\lambda_{2}} \oplus V_{\lambda_{3}} \oplus V_{\lambda_{4}} \text {, }
$$

where

$$
\lambda_{1}=x, \quad \lambda_{2}=-\frac{1}{x}, \quad \lambda_{3}=\frac{1+x}{1-x}, \quad \lambda_{4}=\frac{x-1}{x+1}, \quad \text { and } x=\cot r .
$$

By the same calculation as in the proof of Theorem 1 we know the following:

(1) For each $V_{\lambda_{i}}(i=1,2)$, the distribution $V_{\lambda_{i}} \oplus\{\xi\}_{\mathbf{R}}$ is integrable.

(2) For each $V_{\lambda_{i}}(i=3,4), V_{\lambda_{i}}$ is integrable and every leaf of $V_{\lambda_{i}}$ is a totally geodesic submanifold of the real hypersurface $M$.

Hence, from the argument in the proof of Theorem 1 and from Proposition 2 we obtain the following:

THEOREM 2. Let $M$ be a real hypersurface of $\mathbf{C} P^{n}(n \geqq 2)$. Suppose that the holomorphic distribution $T^{0} M$ satisfies condition $(D)$. Then $M$ is locally congruent to a Hopf hypersurface with constant principal curvatures if and only if every principal foliation $V_{\lambda_{i}}$ of $T^{0} M$ satisfies one of the following two conditions:

(i) The distribution $V_{\lambda_{i}} \oplus\{\xi\}_{\mathbf{R}}$ is integrable.

(ii) $V_{\lambda_{i}}$ is integrable and every leaf of $V_{\lambda_{i}}$ is a totally geodesic submanifold of the real hypersurface $M$.

REMARKS ON THEOREM 2. (1) There does not exist $V_{\lambda_{i}}$ which satisfies the both conditions (i) and (ii) in Theorem 2.

(2) The following result shows that Theorem 2 is no longer true if we omit the condition that every leaf of $V_{\lambda_{i}}$ is a totally geodesic submanifold of the real hypersurface $M$ in (ii).

THEOREM 3. There exist Hopf hypersurfaces $M$ in $\mathbf{C} P^{n}$ with $n \geqq 3$ which satisfy the following properties:

(a) The holomorphic distribution $T^{0} M$ satisfies the decompositon condition (D).

(b) Every principal foliation on $M$ is integrable.

(c) Not every leaf of the principal foliations on $M$ is a totally geodesic submanifold of $M$.

(d) Not every principal curvature of $M$ is locally constant.

Proof. First recall that the Hopf fibration $\pi: S^{2 n+1} \rightarrow \mathbf{C} P^{n}$ from $S^{2 n+1} \equiv\{z \in$ $\left.\mathbf{C}^{n+1} \mid\|z\|=1\right\}$ onto $\mathbf{C} P^{n}$ is a principal fiber bundle with structure group $S^{1} \equiv\left\{e^{i \theta} \mid \theta \in \mathbf{R}\right\}$. For each $z \in S^{2 n+1}$, let $T_{z}^{\prime}$ denote the horizontal subspace of $T_{z} S^{2 n+1}$ consisting of all vectors at $z$ which are orthogonal to $z$ and $i z$. Since $T^{\prime}$ is invariant by the action of $S^{1}, \pi$ induces an isomorphism $\pi_{*}: T_{z}^{\prime} \rightarrow T_{[z]} \mathbf{C} P^{n}$ for any $z \in S^{2 n+1}$, where $[z]=\pi(z)$. 
The Fubini-Study metric $\tilde{g}$ on $\mathbf{C} P^{n}$ of constant holomorphic sectional curvature one is defined as follows: For $\tilde{X}, \tilde{Y} \in T_{[z]} \mathbf{C} P^{n}$, let $z$ be any point in $\pi^{-1}([z]) \cap S^{2 n+1}$ and let $X, Y \in T_{z}^{\prime}$ be the horizontal lifts of $\tilde{X}, \tilde{Y}$ at $z$, then $\tilde{g}_{[z]}(\tilde{X}, \tilde{Y})=4 g_{z}(X, Y)$. The metric $\tilde{g}$ is well defined because if $z^{\prime}$ is also a representation of $[z], z^{\prime}=e^{i \theta} z$ and so $g_{z^{\prime}}\left(e^{i \theta} X, e^{i \theta} Y\right)=$ $g_{z}(X, Y)$.

Let

$$
\begin{aligned}
f_{a, k}(z) & =z_{0}^{2}+\cdots+z_{k}^{2}+a\left(z_{k+1}^{2}+\cdots+z_{n}^{2}\right), \\
z & =\left(z_{0}, z_{1}, \cdots, z_{n}\right) \in \mathbf{C}^{n+1},
\end{aligned}
$$

where $k \geqq 1, n-k \geqq 2$ and $a$ is a positive real number different from 1 . We put

$$
V_{a, k}^{n-1}=\left\{[z]=\left[z_{0}, \cdots, z_{n}\right] \in \mathbf{C} P^{n} \mid f_{a, k}(z)=0\right\}
$$

which is a complex hypersurface of $\mathbf{C} P^{n}$.

For each $[z] \in V_{a, k}^{n-1}$ with $z \in \pi^{-1}([z]) \cap S^{2 n+1}$, the tangent space $T_{[z]} V_{a, k}^{n-1}$ can be identified with

$$
T_{z}=\left\{x \in \mathbf{C}^{n+1} \mid\langle X, z\rangle=\langle X, i z\rangle=\left\langle X, \frac{\partial f_{a, k}}{\partial z}\right\rangle=\left\langle X, i \frac{\partial f_{a, k}}{\partial z}\right\rangle=0\right\},
$$

where $\langle$,$\rangle denotes the Euclidean inner product induced from \mathbf{C}^{n+1}$. The vector field $\xi \equiv$ $\frac{\partial f_{a, k}}{\partial z} /\left(2\left\|\frac{\partial f_{a, k}}{\partial z}\right\|\right)$ can be regarded as a unit normal vector field of $V_{a, k}^{n-1}$ in $\mathbf{C} P^{n}$. For each $X \in T_{[z]} V_{a, k}^{n-1}$, the shape operator $A_{\xi}$ at [z] $\in V_{a, k}^{n-1}$ is given by (cf. [21]):

$$
A_{\xi}(X)=-\frac{1}{2\left\|\frac{\partial f_{a, k}}{\partial z}\right\|} \bar{X}\left(\overline{\frac{\partial^{2} f_{a, k}}{\partial z_{i} \partial z_{j}}}\right)+\zeta \frac{\overline{\partial f_{a, k}}}{\partial z},
$$

where $\bar{w}$ is the complex conjugate of $w,\|\|$ is the Euclidean norm, and

$$
\zeta=\frac{1}{2\left\|\frac{\partial f_{a, k}}{\partial z}\right\|^{3}} \bar{X}\left(\overline{\frac{\partial^{2} f_{a, k}}{\partial z_{i} \partial z_{j}}}\right)\left(\frac{\partial f_{a, k}}{\partial z}\right)^{T} .
$$

We define three subsets $U_{1}, U_{2}, U_{3}$ of $V_{a, k}^{n-1}$ by

$$
\begin{aligned}
& U_{1}=\left\{\left[z_{0}, \cdots, z_{n}\right] \in V_{a, k}^{n-1} \mid z_{k+1}=\cdots=z_{n}=0\right\}, \\
& U_{2}=\left\{\left[z_{0}, \cdots, z_{n}\right] \in V_{a, k}^{n-1} \mid z_{0}=\cdots=z_{k}=0\right\}, \\
& U_{3}=\left\{\left[z_{0}, \cdots, z_{n}\right] \in V_{a, k}^{n-1} \mid \sum_{j=0}^{k}\left\|z_{j}\right\| \neq 0 \text { and } \sum_{i=k+1}^{n}\left\|z_{i}\right\| \neq 0\right\} .
\end{aligned}
$$

Clearly, $U_{3}$ is a dense open subset of $V_{a, k}^{n-1}$ and $U_{1}$ can be regarded as the complex quadric $Q_{k-1} \equiv\left\{\left[z_{0}, \cdots, z_{k}\right] \mid \sum_{j=0}^{k} z_{j}^{2}=0\right\}$ in $\mathbf{C} P^{k}$, which is a totally geodesic complex submanifold of $\mathbf{C} P^{n}$ defined by $\mathbf{C} P^{k} \equiv\left\{[z] \in \mathbf{C} P^{n} \mid z_{k+1}=\cdots=z_{n}=0\right\}$. Similarly, $U_{2}$ can be regarded as a complex quadric in $\mathbf{C} P^{n-k-1} \subset \mathbf{C} P^{n}$.

Now, we determine the eigenvalues of the shape operator together with their multiplicities. 
Case (a): $\quad p \in U_{1}$. Since the group $A\left(\mathbf{C} P^{k}\right)$ of holomorphic isometries of $\mathbf{C} P^{k}$ acts transitively on $Q_{k-1}$ and $A\left(\mathbf{C} P^{k}\right)$ is a subgroup of $A\left(\mathbf{C} P^{n}\right)$, by applying a suitable holomorphic isometry on $\mathbf{C} P^{n}$ if necessary we may assume the homogeneous coordinates of $p$ take the form $[z]_{p}=\left[z_{0}, z_{1}, 0, \cdots, 0\right]$ with $z_{0}, z_{1} \neq 0$. By direct computations we obtain

$$
\begin{aligned}
& T_{[z]_{p}} V_{a, k}^{n-1}=\left\{\left(0,0, v_{2}, \cdots, v_{n}\right) \mid v_{2}, \cdots, v_{n} \in \mathbf{C}\right\} . \\
& \left(\frac{\partial f_{a, k}}{\partial z}\right)_{p}=2\left(z_{0}, z_{1}, 0, \cdots, 0\right), \\
& \left(\frac{\partial^{2} f_{a, k}}{\partial z_{i} \partial z_{j}}\right)_{p}=2\left(\begin{array}{cc}
I_{k+1} & 0 \\
0 & a I_{n-k}
\end{array}\right),
\end{aligned}
$$

where $I_{r}$ is the identity matrix of ordr $r$. From (2.14)-(2.18) we obtain $\zeta=0$ and $\left\|\frac{\partial f_{a, k}}{\partial z}\right\|=2$ at $p$, and also

$$
A_{\xi}(V)=-\frac{1}{2}\left(0,0, \bar{v}_{2}, \cdots, \bar{v}_{k}, a \bar{v}_{k+1}, \cdots, a \bar{v}_{n}\right)
$$

for $V=\left(0,0, v_{2}, \cdots, v_{n}\right) \in T_{[z]_{p}} V_{a, k}^{n-1} \cdot(2.19)$ implies that

$$
\begin{aligned}
& V_{1}=\left\{\left(0,0, r_{2}, \cdots, r_{k}, 0, \cdots, 0\right) \mid r_{2}, \cdots, r_{k} \in \mathbf{R}\right\}, \\
& V_{2}=\left\{i\left(0,0, r_{2}, \cdots, r_{k}, 0, \cdots, 0\right) \mid r_{2}, \cdots, r_{k} \in \mathbf{R}\right\}, \\
& V_{3}=\left\{\left(0, \cdots, 0, r_{k+1}, \cdots, r_{n}\right) \mid r_{k+1}, \cdots, r_{n} \in \mathbf{R}\right\}, \\
& V_{4}=\left\{i\left(0, \cdots, 0, r_{k+1}, \cdots, r_{n}\right) \mid r_{k+1}, \cdots, r_{n} \in \mathbf{R}\right\},
\end{aligned}
$$

are eigenspaces of $A_{\xi}$ with eigenvalues $-1 / 2,1 / 2,-a / 2, a / 2$ and with multiplicities $k$ $1, k-1, n-k, n-k$, respectively. With respect to given orthonormal bases

$$
\left\{e_{2}, \cdots, e_{k}\right\}, \quad\left\{i e_{2}, \cdots, i e_{k}\right\}, \quad\left\{e_{k+1}, \cdots, e_{n}\right\}, \quad\left\{i e_{k+1}, \cdots, i e_{n}\right\}
$$

of $V_{1}, V_{2}, V_{3}, V_{4}$ respectively, we obtain

$$
A_{\xi}=\frac{1}{2}\left(\begin{array}{cccc}
-I_{k-1} & 0 & 0 & 0 \\
0 & I_{n-k} & 0 & 0 \\
0 & 0 & -a I_{n-k} & 0 \\
0 & 0 & 0 & a I_{n-k}
\end{array}\right) .
$$

Therefore, by $A_{J \xi}=J A_{\xi}$ and (2.20), we find

$$
A_{J \xi}=-\frac{1}{2}\left(\begin{array}{cccc}
0 & I_{k-1} & 0 & 0 \\
I_{n-k} & 0 & 0 & 0 \\
0 & 0 & 0 & a I_{n-k} \\
0 & 0 & a I_{n-k} & 0
\end{array}\right) .
$$

(2.20) and (2.21) imply that, for any unit normal vector $\eta=(\cos \theta) \xi+(\sin \theta) J \xi$, we have

$$
A_{\eta}=-\frac{1}{2}\left(\begin{array}{cccc}
\cos \theta I_{k-1} & \sin \theta I_{k-1} & 0 & 0 \\
\sin \theta I_{k-1} & -\cos \theta I_{k-1} & 0 & 0 \\
0 & 0 & a \cos \theta I_{n-k} & a \sin \theta I_{n-k} \\
0 & 0 & a \sin \theta I_{n-k} & -a \cos \theta I_{n-k}
\end{array}\right)
$$


which implies that, for each unit normal vector $\eta, A_{\eta}$ has eigenvalues $-1 / 2,1 / 2,-a / 2, a / 2$ with multiplicities given by $k-1, k-1, n-k, n-k$, respectively.

Case (b): $\quad p \in U_{2}$. In this case, arguments similar to Case (a) show that, for each unit normal vector $\eta$ of $U_{2}$ at $p, A_{\eta}$ has eigenvalues $-1 / 2 a, 1 / 2 a,-1 / 2,1 / 2$ with multiplicities given by $k+1, k+1, n-k-2, n-k-2$, respectively.

Case (c): $p \in U_{3}$. First we claim that there is a holomorphic isometry of $\mathbf{C} \boldsymbol{P}^{n}$ which carries $p$ to a point $\hat{p} \in U_{3}$ whose homogeneous coordinates take the form $[z]_{\hat{p}}=$ $\left[z_{0}, 0, \cdots, 0, z_{n}\right]$ with $z_{0}, z_{n} \neq 0$. This can be seen as follows:

Suppose $p=\left[w_{0}, \cdots, w_{n}\right] \in U_{3}$. Then $\sum_{j=0}^{k}\left\|w_{j}\right\| \neq 0$ and $\sum_{j=k+1}^{n}\left\|w_{j}\right\| \neq 0$. Let $U_{3}^{1} \equiv\left\{\left[v_{0}, \cdots, v_{n}\right] \in U_{3} \mid v_{k+1}=w_{k+1}, \cdots, v_{n}=w_{n}\right\}$ which is the intersection of $U_{3}$ with the $n-k$ linear subspaces of $\mathbf{C} P^{n}$ defined by $z_{j}=w_{j}, j=k+1, \cdots, n$. From $\sum_{j=k+1}^{n}\left\|w_{j}\right\| \neq 0$, it follows that there is a point $q$ in $U_{3}^{1}$ whose homogeneous coordinate takes the form $\left[z_{0}, 0, \cdots, 0, w_{k+1}, \cdots, w_{n}\right]$ with $z_{0} \neq 0$. Since the group $A\left(\mathbf{C} P^{n}\right)$ acts transitively on $\mathbf{C} P^{n}$, there is a holomorphic isometry of $\mathbf{C} P^{n}$ which carries $p$ to $q$. Similarly, there is a holomorphic isometry of $\mathbf{C} P^{n}$ which carries $q$ to a point $\hat{p} \in U_{3}$ whose homogeneous coordinates take the form $[z]_{\hat{p}}=\left[z_{0}, 0, \cdots, 0, z_{n}\right]$ with $z_{0}, z_{n} \neq 0$. Consequently, without loss of generality, we may assume that the homogeneous coordinates of $p$ take the form $\left[z_{0}, 0, \cdots, 0, z_{n}\right]$ with $z_{0}, z_{n} \neq 0$, by applying a suitable holomorphic isometry of $\mathbf{C} P^{n}$ if necessary.

By direct computations we obtain

$$
\begin{aligned}
& T_{[z]_{p}} V_{a, k}^{n-1}=\left\{\left(0, v_{1}, \cdots, v_{n-1}, 0\right) \mid v_{1}, \cdots, v_{n-1} \in \mathbf{C}\right\} . \\
& \left(\frac{\partial^{2} f_{a, k}}{\partial z_{i} \partial z_{j}}\right)_{p}=2\left(\begin{array}{cc}
I_{k+1} & 0 \\
0 & a I_{n-k}
\end{array}\right) .
\end{aligned}
$$

From (2.14), (2.22) and (2.23) we obtain $\zeta=0$ and

$$
A_{\xi}(V)=-\frac{1}{\left\|\frac{\partial f_{a, k}}{\partial z}\right\|}\left(0, \bar{v}_{1}, \cdots, \bar{v}_{k}, a \bar{v}_{k+1}, \cdots, a \bar{v}_{n-1}, 0\right)
$$

for $V=\left(0, v_{1}, \cdots, v_{n-1}, 0\right) \in T_{[z]_{p}} V_{a, k}^{n-1} .(2.24)$ implies that $A_{\xi}$ has eigenvalues $-\left\|\frac{\partial f_{a, k}}{\partial z}\right\|^{-1}$, $\left\|\frac{\partial f_{a, k}}{\partial z}\right\|^{-1},-a\left\|\frac{\partial f_{a, k}}{\partial z}\right\|^{-1}$ and $a\left\|\frac{\partial f_{a, k}}{\partial z}\right\|^{-1}$ with multiplicities $k, k, n-k-1$ and $n-k-1$, respectively. By applying the same argument as in Case (a), we also know that, for each unit normal vector $\eta, A_{\eta}$ has the same eigenvalues and with the same multiplicities as $A_{\xi}$.

Since $k \geqq 1$ and $n-k \geqq 2$, these imply that $U_{3}$ has four distinct principal curvatures and they satisfy the following two properties:

(1) Each eigenvalue of the shape operator of $U_{3}$ with respect to any given unit normal vector $\eta$ is not zero.

(2) The multiplicity of each eigenvalue of $A_{\eta}$ with respect to any unit normal vector $\eta$ constant.

Let $M$ be the real hypersurface in $\mathbf{C} P^{n}$ given by the tube of radius $r(r>0)$ over the complex hypersurface $U_{3}$. Then $M$ has at most five distinct principal curvatures in $\mathbf{C} P^{n}$. 
Moreover, the holomorphic distribution $T^{0} M$ of $M$ satisfies properties (a) and (b) of Theorem 3 (cf. [6]). Furthermore, because $M$ is not in the list of Theorem A, properties (c) and (d) must hold according to Theorem 2 .

\section{Another characterization of Hopf hypersurfaces with constant principal curvatures.}

A unit speed curve $\gamma=\gamma(s)$ in a Riemannian manifold $M$ is called a circle if there exists a field of unit vectors $Y=Y(s)$ along the curve and a constant $\kappa(\geqq 0)$ which satisfy the differential equations: $\nabla_{\dot{\gamma}} \dot{\gamma}=\kappa Y$ and $\nabla_{\dot{\gamma}} Y=-\kappa \dot{\gamma}$, where $\nabla_{\dot{\gamma}}$ denotes the covariant differentiation along $\gamma$ with respect to the Riemannian connection $\nabla$ of $M$. The constant $\kappa$ is called the curvature of the circle. A circle with zero curvature is nothing but a geodesic.

It is well-known that a hypersurface $M^{n}$ in $\mathbf{E}^{n+1}$ is locally an ordinary sphere if and only if all geodesics of $M$ are circles of positive curvature in $\mathbf{E}^{n+1}$. However, there do not exist real hypersurfaces in a nonflat complex space form $\tilde{M}_{n}(c)$ all of whose geodesics are circles in $\tilde{M}_{n}(c)$. This fact shows that the condition that all geodesics of $M$ are circles in $\tilde{M}_{n}(c)$ is too strong for real hypersurfaces $M$ in nonflat complex space forms. So, one shall consider a weaker condition.

THEOREM 4. A connected real hypersurface $M$ of a nonflat complex space form $M_{n}(4 c)$ is a Hopf hypersurface with constant principal curvatures if and only if, at each point $p$ of $M$, there exist orthonormal vectors $v_{1}, v_{2}, \cdots, v_{2 n-2}$ perpendicular to the characteristic vector $\xi$ such that all the geodesics $\gamma_{i}=\gamma_{i}(s)(1 \leqq i \leqq 2 n-2)$ in $M$ with $\gamma_{i}(0)=p$ and $\dot{\gamma}_{i}(0)=v_{i}$ are circles with positive curvature in $\tilde{M}_{n}(4 c)$.

PROOF. When $c>0$, this is done in [3]. So, we only to prove the theorem in the case of $c<0$. Without loss of generality, we may assume $c=-1$.

$\Rightarrow$ We shall prove, for each member in the list of Theorem B case by case that, at each point $p \in M$ there exist $2 n-2$ orthonormal principal curvature vectors $v_{1}, \cdots, v_{2 n-2}$ perpendicular to $\xi$ such that all of the geodesics $\gamma_{i}=\gamma_{i}(s)(1 \leqq i \leqq 2 n-2)$ in $M$ with $\gamma_{i}(0)=p$ and $\dot{\gamma}_{i}(0)=v_{i}$ are circles with positive curvature in $\mathbf{C} H^{n}$.

First, assume $M$ is of type A (i.e., $M$ is of type $A_{0}$, type $A_{1}$ or type $A_{2}$ ). We shall show that $\dot{\gamma}_{i}(s)$ is a principal vector field. Since $\dot{\gamma}_{i}(0)=v_{i}$ is principal, we can set $A \dot{\gamma}_{i}(0)=$ $k_{i} \dot{\gamma}_{i}(0)$. Observe that

$$
\begin{aligned}
\nabla_{\dot{\gamma}_{i}}\left\|A \dot{\gamma}_{i}(s)-k_{i} \dot{\gamma}_{i}(s)\right\|^{2} & =\nabla_{\dot{\gamma}_{i}}\left\langle A \dot{\gamma}_{i}, A \dot{\gamma}_{i}\right\rangle-2 k_{i} \nabla_{\dot{\gamma}_{i}}\left\langle A \dot{\gamma}_{i}, \dot{\gamma}_{i}\right\rangle \\
& =\left\langle\left(\nabla_{\dot{\gamma}_{i}} A\right) \dot{\gamma}_{i}, A \dot{\gamma}_{i}\right\rangle-2 k_{i}\left\langle\left(\nabla_{\dot{\gamma}_{i}} A\right) \dot{\gamma}_{i}, \dot{\gamma}_{i}\right\rangle \\
& =2 \eta\left(\dot{\gamma}_{i}\right)\left\langle\phi \dot{\gamma}_{i}, A \dot{\gamma}_{i}\right\rangle .
\end{aligned}
$$

On the other hand, Proposition B implies

$$
\begin{aligned}
\eta\left(\dot{\gamma}_{i}\right)\left\langle\phi \dot{\gamma}_{i}, A \dot{\gamma}_{i}\right\rangle & =\eta\left(\dot{\gamma}_{i}\right)\left\langle A \phi \dot{\gamma}_{i}, \dot{\gamma}_{i}\right\rangle \\
& =\eta\left(\dot{\gamma}_{i}\right)\left\langle\phi A \dot{\gamma}_{i}, \dot{\gamma}_{i}\right\rangle \\
& =-\eta\left(\dot{\gamma}_{i}\right)\left\langle A \dot{\gamma}_{i}, \phi \dot{\gamma}_{i}\right\rangle .
\end{aligned}
$$


Thus, $\eta\left(\dot{\gamma}_{i}\right)\left\langle\phi \dot{\gamma}_{i}, A \dot{\gamma}_{i}\right\rangle=0$, and hence $\left\|A \dot{\gamma}_{i}(s)-k_{i} \dot{\gamma}_{i}(s)\right\|$ is constant on $\dot{\gamma}_{i}$. Since $A \dot{\gamma}_{i}(0)=$ $k_{i} \dot{\gamma}_{i}(0)$, these imply $A \dot{\gamma}_{i}(s)=k_{i} \dot{\gamma}_{i}(s)$ for any $s$, i.e., $\dot{\gamma}_{i}(s)$ is principal. Combining this with (1.1) and (1.2), we have $\tilde{\nabla}_{\dot{\gamma}_{i}} \dot{\gamma}_{i}=k_{i} N$ and $\tilde{\nabla}_{\dot{\gamma}_{i}} N=-k_{i} \dot{\gamma}_{i}$. Therefore, every geodesic $\gamma_{i}=\gamma_{i}(s)$ in $M$ with $\gamma_{i}(0)=p$ and $\dot{\gamma}_{i}(0)=v_{i}(\perp \xi)$ is a circle of curvature $\left|k_{i}\right|$ in $\mathbf{C} H^{n}$ for each $i \in\{1, \cdots, 2 n-2\}$.

Next, assume $M$ is of type B. Then $T^{0} M$ is decomposed as: $T^{0} M=V_{\tanh (r)} \oplus V_{\operatorname{coth}(r)}$. Let $L_{\tanh (r)}$ and $L_{\operatorname{coth}(r)}$ be leaves of the principal foliations $V_{\tanh (r)}$ and $V_{\operatorname{coth}(r)}$, respectively. From the proof of Theorem 1 we know that each geodesic of $L_{\tanh (r)}\left(\operatorname{resp} . L_{\operatorname{coth}(r)}\right)$ is a geodesic of the real hypersurface $M$ and moreover it is a circle of positive curvature $\tanh (r)$ (resp. $\operatorname{coth}(r))$ in $\mathbf{C H}^{n}$.

$(\Leftarrow)$ Assume that at each point $p$ of $M$ there exist $2 n-2$ orthonormal vectors $v_{1}, v_{2}, \cdots, v_{2 n-2} \perp \xi$ such that the geodesics $\gamma_{i}=\gamma_{i}(s)(1 \leqq i \leq 2 n-2)$ in $M$ with $\gamma_{i}(0)=p$ and $\dot{\gamma}_{i}(0)=v_{i}$ are circles with positive curvature in $\mathbf{C H}^{n}$. These circles satisfy

$$
\tilde{\nabla}_{\dot{\gamma}_{i}}\left(\tilde{\nabla}_{\dot{\gamma}_{i}} \dot{\gamma}_{i}\right)=-k_{i}^{2} \dot{\gamma}_{i},
$$

for some positive constants $k_{i}$, where $\tilde{\nabla}$ is the Riemannian connection of $\mathbf{C H}$.

On the other hand, it follows from (1.1) and (1.2) that

$$
\tilde{\nabla}_{\dot{\gamma}_{i}}\left(\tilde{\nabla}_{\dot{\gamma}_{i}} \dot{\gamma}_{i}\right)=\left\langle\left(\nabla_{\dot{\gamma}_{i}} A\right) \dot{\gamma}_{i}, \dot{\gamma}_{i}\right\rangle N-\left\langle A \dot{\gamma}_{i}, \dot{\gamma}_{i}\right\rangle A \dot{\gamma}_{i} .
$$

By comparing the tangential components of (3.1) and of (3.2) we find

$$
\left\langle A \dot{\gamma}_{i}(s), \dot{\gamma}_{i}(s)\right\rangle A \dot{\gamma}_{i}(s)=k_{i}^{2} \dot{\gamma}_{i}(s),
$$

so we obtain at $s=0$

$$
\left\langle A v_{i}, v_{i}\right\rangle A v_{i}=k_{i}^{2} v_{i} .
$$

Note that $k_{i} \neq 0,1 \leqq i \leqq 2 n-2$. Hence, we have

$$
A v_{i}=k_{i} v_{i} \quad \text { or } \quad A v_{i}=-k_{i} v_{i} \quad \text { for } i=1, \cdots, 2 n-2,
$$

which implies that $\xi$ is principal.

Now, consider the open dense subset $\mathcal{U}$ of $M$ defined by

$$
\begin{array}{r}
\mathcal{U}=\{p \in M \mid \text { the multiplicity of each principal curvature of } \\
\left.M \text { in } \mathbf{C} H^{n} \text { is constant on some neighborhood } \mathcal{V}_{p} \text { of } p\right\} .
\end{array}
$$

Recall that principal curvatures are differentiable on $\mathcal{U}$ and, moreover, the principal curvature vectors can be chosen to be smooth on a sufficiently small neighborhood of each point $p \in \mathcal{U}$. In the following, we shall work on a such fixed neighborhood $\mathcal{V}_{p}$ of $p$.

We divide our discussion into the following two cases:

(I) $\alpha \neq k_{i}$ and $\alpha \neq-k_{i}$ for $\forall i \in\{1, \cdots, 2 n-2\}$ at some point $p \in \mathcal{U}$.

(II) There exists $i \in\{1, \cdots, 2 n-2\}$ such that $\alpha=k_{i}$ or $\alpha=-k_{i}$ holds on $\mathcal{U}$.

Case (I): At the fixed point $p$, the tangent space $T_{p} M$ is decomposed as:

$$
\begin{gathered}
T_{p} M=\{\xi\}_{\mathbf{R}} \oplus\left\{v \in T_{p} M \mid A v=-k_{i_{1}} v\right\} \oplus\left\{v \in T_{p} M \mid A v=k_{i_{1}} v\right\} \oplus \cdots \\
\oplus\left\{v \in T_{p} M \mid A v=-k_{i_{g}} v\right\} \oplus\left\{v \in T_{p} M \mid A v=k_{i_{g}} v\right\}
\end{gathered}
$$


where $0<k_{i_{1}}<k_{i_{2}}<\cdots<k_{i_{g}}$ and $g$ denotes the number of positive distinct $k_{j}, j=$ $1, \cdots, 2 n-2$. Note that each $k_{i_{j}}$ is differentiable on $\mathcal{V}_{p}$.

Now we prove the constancy of $k_{i_{1}}, \cdots, k_{i_{g}}$. It suffices to prove the case of $A v_{i_{j}}=$ $k_{i_{j}} v_{i_{j}}$. By hypothesis we have $v_{i_{j}} k_{i_{j}}=0$ for $j=1, \cdots, g$. Since $A$ is symmetric, we also have

$$
\left\langle\left(\nabla_{v_{i j}} A\right) v_{l}, v_{i_{j}}\right\rangle=\left\langle v_{l},\left(\nabla_{v_{i_{j}}} A\right) v_{i_{j}}\right\rangle, \quad 1 \leqq l \neq i_{j} \leqq 2 n-2 .
$$

In order to apply (3.4), we extend $v_{l}, v_{i_{j}} \in T_{p} M$ to vector fields $V_{l}, V_{i_{j}}$ on some sufficient small neighborhood $\mathcal{W}_{p}\left(\subset \mathcal{V}_{p}\right)$ as follows:

We define $V_{l}$ to be a vector field on $\mathcal{W}_{p}$ satisfying $\left(V_{l}\right)_{p}=v_{l}$ and $V_{l} \perp \xi$. In order to define $V_{i_{j}}$, first we define a smooth vector field $W_{i_{j}}$ on $\mathcal{W}_{p}$ by using the parallel displacement of the vector $v_{i j}$ along geodesics through $p$. We remark that although $W_{i_{j}}$ is not principal on $\mathcal{W}_{p}$ in general, but we have $A W_{i_{j}}=k_{i_{j}} W_{i_{j}}$ along the geodesic $\gamma=\gamma(s)$ with $\gamma(0)=p$ and $\dot{\gamma}(0)=v_{i_{j}}$. We define a vector field $U_{i_{j}}$ on $\mathcal{W}_{p}$ by $U_{i_{j}}=\left(\prod_{\kappa \neq k_{i j}}(A-\kappa I)\right) W_{i_{j}}$, where $\kappa$ runs over the set of all distinct principal curvatures of $M$ except $k_{i_{j}}$. Then

$$
\begin{aligned}
A U_{i_{j}} & =A\left(\prod_{\kappa \neq k_{i_{j}}}(A-\kappa I)\right) W_{i_{j}}=\left(\prod_{\kappa \neq k_{i_{j}}}(A-\kappa I)\right) A\left(V_{k_{i_{j}}} \text { component of } W_{i_{j}}\right) \\
& =k_{i_{j}} U_{i_{j}} \neq 0
\end{aligned}
$$

on $\mathcal{W}_{p}$. We put $V_{i_{j}}=U_{i_{j}} /\left\|U_{i_{j}}\right\|$. Our construction guarantees that $A V_{i_{j}}=k_{i_{j}} V_{i_{j}},\left(V_{i_{j}}\right)_{p}=$ $v_{i_{j}}$ and the integral curve of $V_{i_{j}}$ through the point $p$ is a geodesic of $M$. In particular, we obtain $\left(\nabla_{V_{i_{j}}} V_{i_{j}}\right)_{p}=0$.

Since the equation (1.7) of Codazzi implies

$$
\left\langle\left(\nabla_{X} A\right) Y, Z\right\rangle=\left\langle\left(\nabla_{Y} A\right) X, Z\right\rangle \quad \text { for } \forall X, Y, Z \perp \xi,
$$

at the point $p$, we find

$$
\begin{aligned}
\text { The left hand side of (3.4)) } & =\left\langle\left(\nabla_{v_{l}} A\right) v_{i_{j}}, v_{i_{j}}\right\rangle \\
& =\left\langle\left(\nabla_{V_{l}} A\right) V_{i_{j}}, V_{i_{j}}\right\rangle_{p} \\
& =\left\langle\left(V_{l} k_{i_{j}}\right) V_{i_{j}}+\left(k_{i_{j}} I-A\right) \nabla_{V_{l}} V_{i_{j}}, V_{i_{j}}\right\rangle_{p} \\
& =v_{l} k_{i_{j}} .
\end{aligned}
$$

Similarly we get

$$
\begin{aligned}
\text { (The right hand side of (3.4)) } & =\left\langle V_{l},\left(\nabla_{V_{i_{j}}} A\right) V_{i_{j}}\right\rangle_{p} \\
& =\left\langle V_{l}, \nabla_{V_{i_{j}}}\left(k_{i_{j}} V_{i_{j}}\right)-A \nabla_{V_{i_{j}}} V_{i_{j}}\right\rangle_{p} \\
& =\left\langle v_{l},\left(v_{i_{j}} k_{i_{j}}\right) v_{i_{j}}\right\rangle=0 .
\end{aligned}
$$

These imply that $X k_{i_{j}}=0$ for any $X \in T_{p} M$ which is perpendicular to $\xi$.

Next, we prove $\xi k_{i_{j}}=0$. We divide the proof into two cases: 
Case $\left(\mathrm{I}_{a}\right): \quad 2 k_{i_{j}}-\alpha \neq 0$ at a point $p \in \mathcal{U}$. In this case, we obtain from Proposition $\mathrm{A}$ that

$$
A \phi V_{i_{j}}=\left(\frac{\alpha k_{i_{j}}-2}{2 k_{i_{j}}-\alpha}\right) \phi V_{i_{j}}
$$

on some neighborhood $\mathcal{W}_{p}\left(\subset \mathcal{V}_{p}\right)$ of $p$. This, together with (1.5), yields

$$
\begin{aligned}
\left(\nabla_{\xi} A\right) V_{i_{j}}-\left(\nabla_{V_{i_{j}}} A\right) \xi & =\nabla_{\xi}\left(A V_{i_{j}}\right)-A \nabla_{\xi} V_{i_{j}}-\nabla_{V_{i_{j}}}(\alpha \xi)+A \nabla_{V_{i_{j}}} \xi \\
& =\nabla_{\xi}\left(k_{i_{j}} V_{i_{j}}\right)-A \nabla_{\xi} V_{i_{j}}-\alpha \phi A V_{i_{j}}+A \phi A V_{i_{j}} \\
& =\left(\xi k_{i_{j}}\right) V_{i_{j}}+\left(k_{i_{j}} I-A\right) \nabla_{\xi} V_{i_{j}}-k_{i_{j}}\left(\alpha-\frac{\alpha k_{i_{j}}-2}{2 k_{i_{j}}-\alpha}\right) \phi V_{i_{j}} .
\end{aligned}
$$

On the other hand, (1.7) yields

$$
\left(\nabla_{\xi} A\right) V_{i_{j}}-\left(\nabla_{V_{i_{j}}} A\right) \xi=-\phi V_{i_{j}}
$$

By combining these two equations we find $\xi k_{i_{j}}=0$. Consequently, $k_{i_{j}}$ is constant on $\mathcal{W}_{p}$.

Case $\left(\mathrm{I}_{b}\right): 2 k_{i_{j}}-\alpha=0$ on $\mathcal{U}$. In this case, $k_{i_{j}}=\alpha / 2$ is locally constant. This case occurs only when $M$ is a type $\left(A_{0}\right)$ hypersurface, i.e., a horosphere.

Case (II): Let $p$ be a point in $\mathcal{U}$. Recall that the multiplicity of each principal curvature of $M$ is constant on some neighborhood $\mathcal{V}_{p}$ of $p$. Since $\alpha$ is locally constant, the assumption $\alpha=k_{i}$ or $\alpha=-k_{i}$ implies that $k_{i}$ is locally constant. The constancy of the other distinct $k_{i}$ follows from the same discussion as in Case (I).

Consequently, we know that every principal curvature of $M$ is locally constant on the open dense subset $\mathcal{U}$ of $M$. Since $M$ is assumed to be connected, each principal curvature is constant on $M$. Finally we remark that Case (II) occurs only when $M$ is a type $B$ hypersurface with radius $r=\log _{e}(2+\sqrt{3})$ (cf. [12]).

The following result is an immediate consequence of Theorem 4.

COROLLARY. Let $M$ be a connected real hypersurface of a nonflat complex space form $M_{n}(4 c)$. Then $M$ is locally congruent to one of type $A_{1}$ hypersurface with radius $r(0<r<$ $\pi / 2$ ) or a type $A_{2}$ hypersurface with radius $r=\pi / 4$ in the list of Theorem $A$; or one of type $A_{0}$ hypersurface with radius $r(0<r<\infty)$ and type $A_{1}$ hypersurface with radius $r(0<r<\infty)$ in the list of Theorem $B$ if and only if, at each point $p$ of $M$, there exist orthonormal vectors $v_{1}, \cdots, v_{2 n-2}$ orthogonal to the characteristic vector $\xi$ such that all the geodesics $\gamma_{i}=\gamma_{i}(s)(1 \leqq i \leqq 2 n-2)$ on $M$ with $\gamma_{i}(0)=p$ and $\dot{\gamma}_{i}(0)=v_{i}$ are circles in $\tilde{M}_{n}(4 c)$ with the same positive curvature.

REMARKS ON THEOREM 4. (1) In Theorem 4 we do not need to assume that $\left\{v_{1}, v_{2}, \cdots, v_{2 n-2}\right\}$ is a local fields of orthonormal frames. However, for each member $M$ in the lists of Theorem A and Theorem B, one can take a local field of orthonormal frames in $M$ satisfying the hypothesis of Theorem 4.

(2) Every circle in $\mathrm{CP}^{n}(4)$ (resp. $\mathrm{CH}^{n}(-4)$ ) given in Theorem 4 is a simple curve lying in some totally real totally geodesic $\mathbf{R} P^{2}(1)$ (resp. $\mathbf{R} H^{2}(-1)$ ). However, the feature of 
circles in $\mathbf{C} H^{n}(-4)$ is more complicated than circles in $\mathbf{C} P^{n}(4)$. In case of $\mathbf{C} P^{n}(4)$ every circle given in Theorem 4 is closed. On the other hand, in the case of $\mathrm{CH}^{n}(-4)$ a circle given in Theorem 4 is closed if and only if its curvature is greater than 1 (see [1], [2] for details).

\section{References}

[ 1 ] T. AdACHI, S. MAedA and S. UdAGAWA, Circles in a complex projective space, Osaka, J. Math. 32 (1995), 709-719.

[ 2 ] T. ADACHI and S. MAEDA, Global behaviors of circles in a complex hyperbolic space, Tsukuba J. Math. 21 (1997), 29-42.

[ 3 ] T. ADACHI, M. Kimura and S. MAEDA, A characterization of all homogeneous real hypersurfaces in a complex projective space by observing the extrinsic shape of geodesics, Arch. Math. 73 (1999), 303-310.

[ 4 ] J. BERNDT, Real hypersurfaces with constant principal curvatures in complex hyperbolic space, J. Reine Angew. Math. 395 (1989), 132-141.

[ 5 ] E. CARTAN, Familles de surfaces isoparamétriques dans les espaces á courbure constante, Ann. Math. Pura Appl. (4) 17 (1938), 177-191.

[6] T. E. CECIL and P. J. RYAN, Focal sets and real hypersurfaces in complex projective space, Trans. Amer. Math. Soc. 269 (1982), 481-499.

[7] J. DORFMEISTER and E. NEHER, An algebraic approach to isoparametric hypersurfaces in spheres I, II, Tôhoku Math. J. (2) 35 (1983), 187-224; 225-247.

[ 8 ] D. FERUS, H. KARCHER and H. F. MÜNZNER, Cliffordalgebren und neue isoparametrische Hyperflächen, Math. Z. 177 (1981), 479-502.

[ 9 ] M. KIMURA, Real hypersurfaces and complex submanifolds in complex projective space, Trans. Amer. Math. Soc. 296 (1986), 137-149.

[10] M. KimURA and S. MAEDA, On real hypersurfaces of a complex projective space, Math. Z. 202 (1989), 299-312.

[11] R. MIYAOKA, The homogeneity of isoparametric hypersurfaces with six principal curvatures, Preprint TitechMath. 80 (1998).

[12] S. MONTIEL, Real hypersurfaces of a complex hyperbolic space, J. Math. Soc. Japan 37 (1985), 515-535.

[13] H. F. MÜNZNER, Isoparametrische Hyperflächen in Sphären I, II, Math. Ann. 251 (1980), 57-71; 256 (1981), 215-232.

[14] R. Niebergall and P. J. RyAn, Real hypersurfaces in complex space forms, Tight and Taut Submanifolds, (eds. T. E. CECIL and S. S. CHERN), Cambridge University Press (1998), 233-305.

[15] K. Nomizu, Elie Cartan's work on isoparametric families of hypersurfaces, Differ. Geom., Proc. Symp. Pure Math. 27, Part 1, (1975), 191-200.

[16] K. OGIUE, Differential geometry of Kaehler submanifolds, Adv. in Math. 13 (1974), 73-114.

[17] H. OZEKI and M. TAKEUCHI, On some types of isoparametric hypersurfaces in spheres I, II, Tôhoku Math J. (2) 27 (1975), 515-559; 28 (1976), 7-55.

[18] J. SAITOU, Real hypersurfaces in a complex hyperbolic space with three constant principal curvatures, Tsukuba J. Math. 23 (1999), 353-367.

[19] R. TAKAGI, On homogeneous real hypersurfaces in a complex projective space, Osaka J. Math. 10 (1973), 495-506.

[20] R. TAKAGI, Real hypersurfaces in a complex projective space with constant principal curvatures I, II, J. Math. Soc. Japan 27 (1975), 43-53; 507-516.

[21] A. VitTER, On the curvature of complex hypersurfaces, Indiana Univ. Math. J. 23 (1974), 813-826. 
Present Addresses:

BANG-YEN CHEN

Department of Mathematics, Michigan State University,

EAST LANSING, MI 48824-1027, U.S.A.

e-mail: bychen@math.msu.edu

SADAHIRO MAEDA

DePaRTMENT OF MATHEMATICS, Shimane Univeresity,

MATSUE, 690-8504 JAPAN.

e-mail: smaeda@math.shimane-u.ac.jp 